\title{
Role of carbohydrate response element- binding protein (ChREBP) in generating an aerobic metabolic phenotype and in breast cancer progression
}

\author{
R E Airley ${ }^{\star}, 1$, P McHugh ${ }^{1}$, A R Evans ${ }^{2}$, B Harris ${ }^{3}$, L Winchester ${ }^{3}$, F M Buffa ${ }^{3}$, W Al-Tameemi ${ }^{1}$, R Leek ${ }^{4}$ \\ and A L Harris ${ }^{3}$
}

${ }^{1}$ Division of Pharmacy, University of Huddersfield, Huddersfield HD1 3DH, UK; ${ }^{2}$ School of Pharmacy and Biomolecular Sciences, Liverpool John Moores University, Liverpool L3 3AF, UK; ${ }^{3}$ Department of Oncology, Weatherall Institute for Molecular Medicine, University of Oxford, Oxford OX3 7DQ, UK and ${ }^{4}$ Nuffield Department of Clinical Laboratory Science, University of Oxford, Oxford OX3 7DQ, UK

Background: The lipogenic transcription factor carbohydrate response element-binding protein (ChREBP) may play a key role in malignant progression of breast cancer by allowing metabolic adaptations to take place in response to changes in oxygenation.

Methods: Immunohistochemical analysis of ChREBP was carried out in human breast tumour tissue microarrays representative of malignant progression from normal breast through to metastatic cancer. The ChREBP protein and mRNA expressions were then analysed in a series of breast cancers for correlative analysis with common and breast-specific hypoxia signatures, and survival.

Results: In invasive ductal carcinoma, ChREBP correlated significantly with mean 'downregulated' hypoxia scores $(r=0.3, P<0.015, n=67)$ and in two distinct breast progression arrays, ChREBP protein also increased with malignant progression $(P<0.001)$. However, bioinformatic analysis of a large data set $(2136$ cases) revealed an apparent reversal in the relationship between ChREBP mRNA level and clinical outcome - not only being significantly correlated with increased survival (log rank $P<0.001)$, but also downregulated in malignant tissue compared with adjacent normal tissue.

Conclusion: The ChREBP expression may be reflective of an aerobic metabolic phenotype that may conflict with hypoxia-induced signalling but provide a mechanism for growth at the oxygenated edge of the tumours.

The carbohydrate response element-binding protein (ChREBP) is a glucose-regulated basic helix-loop-helix (bHLH) transcription factor that acts as a key regulator of enzymes involved in fatty acid synthesis. In response to increased glucose levels, ChREBP undergoes dephosphorylation steps that allow translocation from the cytoplasm to the nucleus where, in association with its binding partner MLX (Max-like interacting protein), it binds to the carbohydrate response element of lipogenic genes (Uyeda et al, 2002; Dentin et al, 2005b; Postic et al, 2007).
Evidence indicates that ChREBP may have a role in cancer pathology and the mechanisms associated with tumourigenesis. In particular, a link between ChREBP and the suppression of p53-induced cell cycle arrest has been revealed in transformed cells that takes place via the reprogramming of metabolism to favour aerobic glycolysis (Tong et al, 2009), an established hallmark of tumour metabolism (Airley and Mobasheri, 2007; Annibaldi and Widmann, 2010; Scatena et al, 2010; Bensinger and Christofk, 2012). Furthermore, genomic analysis of ChREBP target gene 
expression in the hepatoma HEPG2 cell line shows a dual association with pathways associated with the tumour metabolic phenotype as well as with malignant progression, such as differentiation and motility (Jeong et al, 2011).

The metabolic shift from one that is dominated by oxidative phosphorylation to that of increased glycolytic flux is manifested by increased glucose flux and often overexpression of the hypoxiainducible factor-1 (HIF-1)- regulated facilitative glucose transporter Glut-1, alongside the associated upregulation of glycolytic enzymes (Airley and Mobasheri, 2007). Metabolomic analysis also indicates that Glut-1 facilitates a HIF-1-independent rise in the production of metabolites associated with phospholipid metabolism and cell turnover, for example phosphatidyl choline (Evans et al, 2008). The sequence homology between the ChRE and the hypoxia response element (HRE) in the promoter of HIF-1 target genes, meanwhile, suggests that there may be a cross-talk or at least coordination of ChREBP and HIF-1-regulated pathways within the spatially and temporally heterogeneous tumour microenvironment (Dang et al, 1997).

So far, much of the work surrounding ChREBP has focussed on its function as a liver transcription factor, its activation by glucose metabolites and role in the regulation of lipogenesis. This may have implications for breast cancer pathology. First, breast cancer is mechanistically and epidemiologically linked with obesity (Calle and Kaaks, 2004; Lorincz and Sukumar, 2006), suggesting a causal link with deregulated lipid metabolism. Second, glycolytic and lipogenic pathways may behave as integrated features of tumour metabolism brought about via loss of p53, activation of Akt and changes in the expression and functionality of lactate dehydrogenase (Young and Anderson, 2008).

The aim of this study was to investigate ChREBP expression in breast cancer and how this may be associated with Glut-1 expression and malignant progression in the context of published genomic analysis of the hypoxic tumour microenvironment.

\section{MATERIALS AND METHODS}

Materials. Unless stated otherwise, all reagents were obtained from Sigma (Dorset, UK). Histology glass wear and mounting medium were purchased from Thermo Scientific (Loughborough, UK). Cell lines were obtained from ATCC (Teddington, UK) (LGC standards) and had been authenticated according to in-house procedures.

Tissue microarrays. Tissue microarrays used in this study included the Accumax (Cepheid UK Ltd, Stretton, UK) (A712) breast cancer test array that carried samples from 12 cases of breast cancer paired with normal adjacent tissue. In addition, two separate breast cancer progression arrays were also used: the Cybrdi Inc (Rockville, MD, USA, CC08-00-005) array that contains 71 individual cases of adenosis, fibroadenoma, tumour to metastasis, and the Biomax (Insight Biotechnology, Wembley, Middlesex, UK, BR2082) array that contains a total of 206 cases made up of 32 samples of metastatic carcinoma, 68 invasive ductal carcinoma, 22 each of lobular carcinoma and intraductal carcinoma, 4 each of squamous cell carcinoma and lobular carcinoma in situ, 8 fibroadenoma, 16 each of hyperplasia and inflammation, 10 adjacent normal tissue and 6 normal tissue. Information on clinical and pathological characteristics as well as ethical considerations is available on the website of, or upon request from, the commercial tissue microarray suppliers. These studies were carried out after approval by the institutional ethical review committee (University of Huddersfield, Huddersfield, USA).

Immunohistochemistry. Immunohistochemistry was carried out as per established protocols, with primary antibody steps consisting of a 1:100 dilution of rabbit anti-Glut-1 (Alpha Diagnostic International, San Antonio, TX, USA) or a 1:400 dilution of rabbit anti-ChREBP (Abcam, Cambridge, UK). For ChREBP immunostaining, antigen retrieval was carried out by boiling slides for $20 \mathrm{~min}$ in $10 \mathrm{~mm}$ citrate buffer. A commercially available kit (Menorini Diagnostics, Reading, UK) that consists of a universal rabbit/mouse secondary probe and a horseradish peroxidase or alkaline phosphatase enzyme polymer conjugate plus chromogen was used for visualisation.

Antibody specificity. The specificity of the rabbit anti-ChREBP used for the immunohistochemical analysis of the tissue microarrays was determined by western analysis of a ChREBP knockdown cell line generated from MCF-7 cells. This revealed a sharp decrease in expression in early passages with a return to wild-type levels within five passages. This apparent reversal of knockdown was confirmed by qRT-PCR analysis (shown in Supplementary Figure S1). Although this confirms the specificity of the antibody, further work will be needed to determine whether the changes in ChREBP expression relates to a technical problem with the generation of the cell lines or is a biological adaptation to reduced ChREBP expression.

The ChREBP knockdown MCF-7-derived cell line and subsequent western and qRT-PCR analyses were carried out.

Generation of ChREBP knockdown MCF7-derived cell line. A ChREBP knockdown cell line and scrambled vector control were generated using reagents obtained from Santa Cruz Biotechnology (Dallas, TX, USA). MCF7 cells were transfected with CHREBP shRNA (h) lentiviral particles (sc-38617-V) using Polybrene (Sigma) as per the manufacturer's protocol. These were used alongside control shRNA lentiviral particles (sc-108080). Stable clones were selected after placing in DMEM containing $2 \mu \mathrm{g} \mathrm{ml}^{-1}$ puromycin (Santa Cruz Biotechnology).

Western blot. Cell lysates were prepared according to standard protocols and the equivalent to $20 \mu \mathrm{g}$ protein samples was run on NuPAGETM Novex electrophoresis pre-cast gels (Invitrogen, Life Technologies, Paisley, UK) followed by transfer onto a PVDF transfer membrane of pore size $0.45 \mu \mathrm{m}$ (Immobilon-P Polyvinylidine difluoride, Millipore, Watford, UK). Membranes were blocked with $1 \%(\mathrm{w} / \mathrm{v})$ casein before overnight incubation at $4{ }^{\circ} \mathrm{C}$ with rabbit anti-ChREBP diluted with SignalBoostImmunoreaction Enhancer primary antibody solution (Merck Chemicals, Watford, UK) according to the manufacturer's instructions. The membranes were visualised using an Odyssey Infra-red Imaging system (Li-Cor, Lincoln, NE, USA), using $\beta$-actin antibody as a loading control.

qRT-PCR analysis. Total RNA was extracted using Tri Reagent (Sigma) as per the manufacturer's instructions. The quantities and qualities of RNA from each sample were assessed by gel agarose electrophoresis as well as spectrophotometry using a NanoDrop 2000 (Thermo Scientific). Then, $1 \mu \mathrm{g}$ of RNA was incubated in a total volume of $10 \mu \mathrm{l}$ containing $1 \mu \mathrm{l}$ of $10 \times$ DNase I reaction buffer and $1 \mu \mathrm{l}\left(1 \mathrm{U} \mu \mathrm{l}^{-1}\right)$ of DNase I at $37^{\circ} \mathrm{C}$ for $30 \mathrm{~min}$ with subsequent inactivation at $65^{\circ} \mathrm{C}$ for 15 min with $1 \mu \mathrm{l} 25 \mathrm{~mm}$ EDTA. The cDNA was synthesised in a $20 \mu \mathrm{l}$ volume with oligo(dT)20 (Invitrogen) and SuperScript III First-Strand Synthesis SuperMix (Invitrogen). The reactions were incubated at $50{ }^{\circ} \mathrm{C}$ for $1 \mathrm{~h}$ and then inactivated by heating at $70{ }^{\circ} \mathrm{C}$ for $15 \mathrm{~min}$. The RNase $\mathrm{H}$ (Invitrogen) was added, $1 \mu \mathrm{l}\left(1 \mathrm{U} \mu \mathrm{l}^{-1}\right)$ to each preparation, and incubated for $20 \mathrm{~min}$ at $37^{\circ} \mathrm{C}$.

In each qRT-PCR reaction, $5 \mathrm{ng}$ of template cDNA was used in a total volume of $15 \mu \mathrm{l}$ containing water, $7.5 \mu \mathrm{l}$ of iTaq Universal SYBR Green 2x Supermix (Bio-Rad, Herts, UK) and the appropriate primer set (Eurofins MWG Operon, Ebersberg, Germany; see Supplementary Information for primer sequences) at a final concentration of $100 \mathrm{~nm}$. The qRT-PCR assays were 
performed on a Bio-Rad CFX96 Touch Real-Time PCR Detection System using the following PCR conditions: $15 \mathrm{~min}$ at $95^{\circ} \mathrm{C}$ followed by 50 cycles of $15 \mathrm{~s}$ denaturing at $95^{\circ} \mathrm{C}$ and $60 \mathrm{~s}$ annealing at $60{ }^{\circ} \mathrm{C}$. A reverse transcriptase negative control was used to assess whether any residual genomic DNA remained and a no-template control was included to examine primer-dimer formation. The qRTPCR experiments were excluded for analysis if the reaction efficiency was outside the range of $0.9-1.1$ or if the $r^{2}$ value of the linear regression was $<0.95$. For the $\mathrm{qPCR}$ analysis, the relative normalised expression $(\Delta \Delta \mathrm{Cq})$ method was determined using $R P L 30$ as a reference gene. The normalised data from the various cell lines were analysed using the Bio-Rad CFX manager software 3.0 and fold change determined.

Semiquantitative and statistical analysis. Semiquantitative scoring was carried out as in previous studies (Evans et al, 2008) where, briefly, tissue microarray sample 'spots' were viewed at low magnification $(\times 200)$ and an overall score assigned according to intensity and area of immunostaining staining. Supplementary Figure S2 shows representative examples of sample scoring as follows: 0 , no; 1 , light; 2, moderate; and 3, heavy staining. To determine interobserver variation, the first 30 samples of the Biomax (BR2082) array were scored by two observers (REA and WA-T) (ChREBP $r=0.854, P<0001, n=30$ ); the intraobserver variation was measured using scores made by the primary observer (WA-T) on the Biomax (BR2082) array 3 weeks apart (Spearman's rank correlation: (Glut-1) $r=0.843, P<0.001, n=32$ (ChREBP) $r=0.952, P<0.001, n=32)$.

Bioinformatic analysis. The derivation of a common hypoxia signature or 'hypoxia metagene', together with a breast cancerspecific hypoxia signature, has been described previously and may be used to provide both prognostic information in the clinic. It also provides data that allow us to integrate gene function and derive further hypotheses with which to investigate how pathways directed by hypoxia are associated with deregulated metabolism within the tumour microenvironment (Favaro et al, 2011; Buffa et al, 2011). These have been used to compile up- and downregulated hypoxia gene signatures using in vitro and in vivo data sets that charted changes in the expression of these genes in hypoxic conditions alongside the involvement of hypoxia-inducible factor HIF-1. A large number, although not all, of these hypoxiaregulated genes are HIF-1 target genes (Buffa et al, 2010). The Oxf-IJB breast cancer series and other published data sets were used for expression analysis and have been described previously (Buffa et al, 2011). For comparison, this included the Curtis breast cancer series of 2136 breast tumours (Curtis et al, 2012) via Oncomine (Compendia Biosciences, Ann Arbor, MI, USA).

\section{RESULTS}

ChREBP immunohistochemistry in tissue microarrays. Immunohistochemical profiling of ChREBP protein expression in tissue microarrays was carried out to determine whether there is differential expression in breast tumours of mixed pathologies relative to adjacent nonmalignant breast tissues. The overexpression of Glut-1 observed in breast and other cancers (Avril et al, 2001; Airley et al, 2010) may provide glucose metabolites with which to activate ChREBP signalling, and therefore we also carried out Glut-1 immunohistochemical analysis in the same tissue microarrays to observe any colocalisation and correlation between Glut-1 and ChREBP. In general, ChREBP immunostaining was found in the cytoplasm and the nucleus. The subcellular location in the nucleus is because of dephosphorylated (active) ChREBP, wherein it is capable of forming a DNA-binding complex (Ma et al, 2005).

Initially, we used a low-density tissue microarray (Accumax A712) to look for any differences in the pattern of ChREBP expression between breast tumours and adjacent normal tissue that would allow preliminary assessment of ChREBP as a biomarker of malignancy in this tissue type (Figure 1). The data from this array

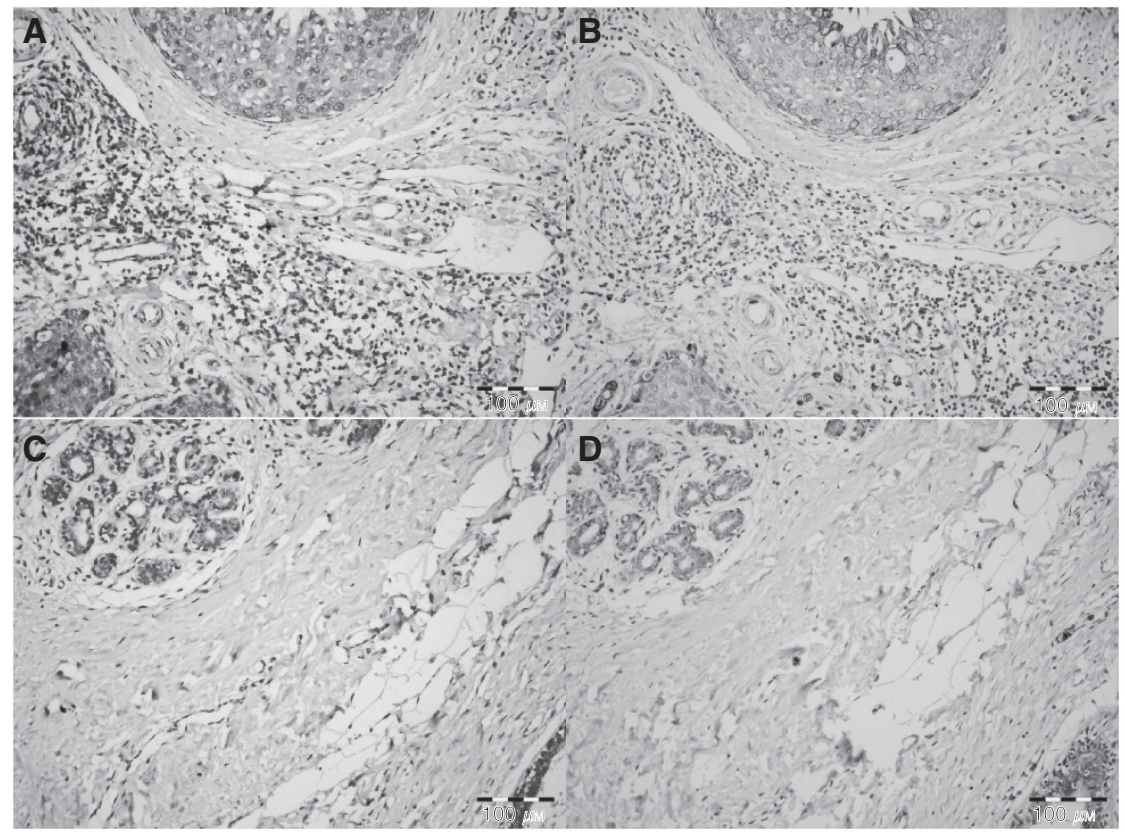

Figure 1. Immunohistochemical staining carried out according to the area and intensity of staining $(0$, absent; 1 , light; 2 , moderate; 3 , heavy staining. (A) The ChREBP staining (Fast Red, pink) in malignant breast compared with an absence of staining in normal adjacent tissue (B) in a test issue microarray (Accumax A712). In cases where normal tissue stained positively for ChREBP, this was at a lower level in both area and intensity. At places where this took place, Glut-1 (DAB, brown) in serial sections was similarly localised ( $C$ and $\mathbf{D}$ ), whether in duct or stromal tissue (shown by arrows). Scale bar $=100 \mu \mathrm{m}$. The full colour version of this figure is available at British Journal of Cancer online. 
showed 5 out of 12 cases clearly expressing ChREBP protein in malignant but not adjacent normal tissue, but 7 out of 12 cases stained positive for ChREBP in both, although the ChREBP staining was at a lower level in normal tissue when present. There was a similarity in localisation between ChREBP and Glut-1 expression in serial sections found in areas of both normal and malignant breast tissue.

To reveal a possible link between ChREBP protein and the histopathology of malignant progression, we used two commercially available breast progression arrays that provided us with samples from two independent series of breast cancer cases. Figure 2 shows representative ChREBP immunostaining in the Cybrdi (CC08-00-005) breast progression array, where staining scores showed a clear trend for increasing with the malignant progression as defined by histopathological diagnosis. Although nonmalignant and inflammatory or hyperplastic tissue tended not to express ChREBP and, if so, at low levels, malignant, infiltrating and metastatic breast tumour tissue was virtually always associated with moderate or heavy ChREBP staining. A similar trend was seen with the Biomax (BR2082) array that also carried a progression of breast histopathologies but with a larger series of cases. In this series, moderate or heavy ChREBP staining was only apparent in invasive tumours, whereas normal tissue, nonmalignant hyperplasia and carcinoma in situ, in all but 2 cases, showed no ChREBP staining at all. Furthermore, Glut-1 and ChREBP scores were significantly correlated (Spearman's rank correlation: $r=461$, $P<0.001, n=207)$. Categorisation into normal, benign hyperplastic/inflammatory, in situ (localised), invasive and metastatic histopathology produced a statistically significant increase in mean immunohistochemical score in both the Cybrdi and Accumax progression arrays, although a significant reverse trend was observed when considering mean ChREBP mRNA expression and malignant progression by similar histopathological designation in the Curtis series of breast cancer cases (2136 patients) via Oncomine (Figure 3A).

Clinical correlates of ChREBP protein and mRNA expression with survival. Kaplan-Meier analysis did not show a significant relationship between grouped ChREBP protein scores in the Oxf-IJB breast cancer series with either overall $(P=0.1)$ or relapsefree $(P=0.6)$ survival in this cohort of patients. However, an analysis of survival data from the Curtis series showed ChREBP mRNA expression to not only be significantly correlated with increased survival (log rank $P<0.001$; MLXIPL ILMN_1722073; Figure $3 \mathrm{~B}$ ) but also show a trend for decreased expression with grade and an overexpression in normal adjacent tissue and benign cases relative to malignant tissue (illustrated by heat maps generated via Oncomine, see Supplementary Slides). A similar trend for increased survival was observed with the ChREBP reporter MLXIPL ILMN_2399919 but this was not significant $(P=0.091)$. When stratified by molecular subtype, there remained a significant increased likelihood of survival with high ChREBP (MLXIPL ILMN_1722073) mRNA expression in oestrogen receptor (ER)-positive cases (log rank $P=0.014$ ), ErbB2-negative (log rank $P=0.004)$ and nontriple-negative (log rank $P<0.001)$ breast cancer. A differential analysis of ChREBP expression by ER status via Oncomine also revealed a significant overexpression (MLXIPL ILMN_1722073 1.082-fold change, $t$-test: $6.219, P=4.02 \mathrm{E}-10$; ILMN_2399919, $t$-test: $4.629, P=2.17 \mathrm{E}-6)$ in a subgroup of ER-positive invasive ductal breast carcinoma within the Curtis breast series.

Clinical correlates of ChREBP protein and mRNA expression with hypoxia. Analysis of the relationship between ChREBP expression and the breast hypoxia gene signature revealed a significant correlation between ChREBP protein and mean 'downregulated' hypoxia scores $(r=0.3, P=0.014, n=67)$, which was weaker but consistent at mRNA level $(r=0.134, P=0.2$, $n=67$ ) There was also a significant anticorrelation between ChREBP protein and median 'upregulated' hypoxia scores $(r=-0.246, P=0.04, n=67)$. There was, however, no relationship to scores derived from either the up- or downregulated lactate signature described by Chen et al (2008).

The ChREBP mRNA levels mined from an extended series of 232 cases analysed using an Illumina (San Diego, CA, USA) platform correlated significantly with both mean and median

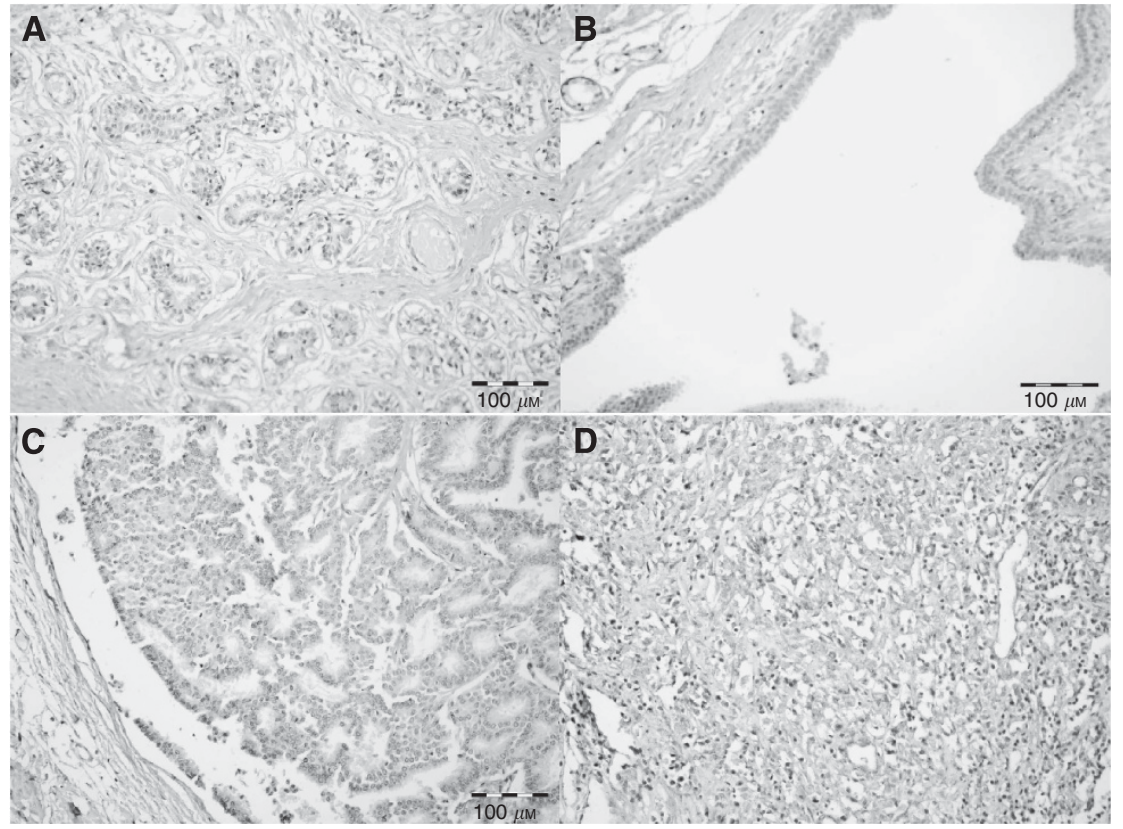

Figure 2. The ChREBP protein staining (Fast Red) in a human breast cancer progression array (Cybrdi CC08-00-005), showing a clear trend for increased staining score with malignant progression from normal breast to metastatic breast cancer. (A) Normal breast (score 0); (B) mild atypical ductal hyperplasia (score 1); (C) intraductal carcinoma (score 2); and (D) metastatic nonspecific infiltrating ductal carcinoma (score 3). Scale bar $=100 \mu \mathrm{m}$. 

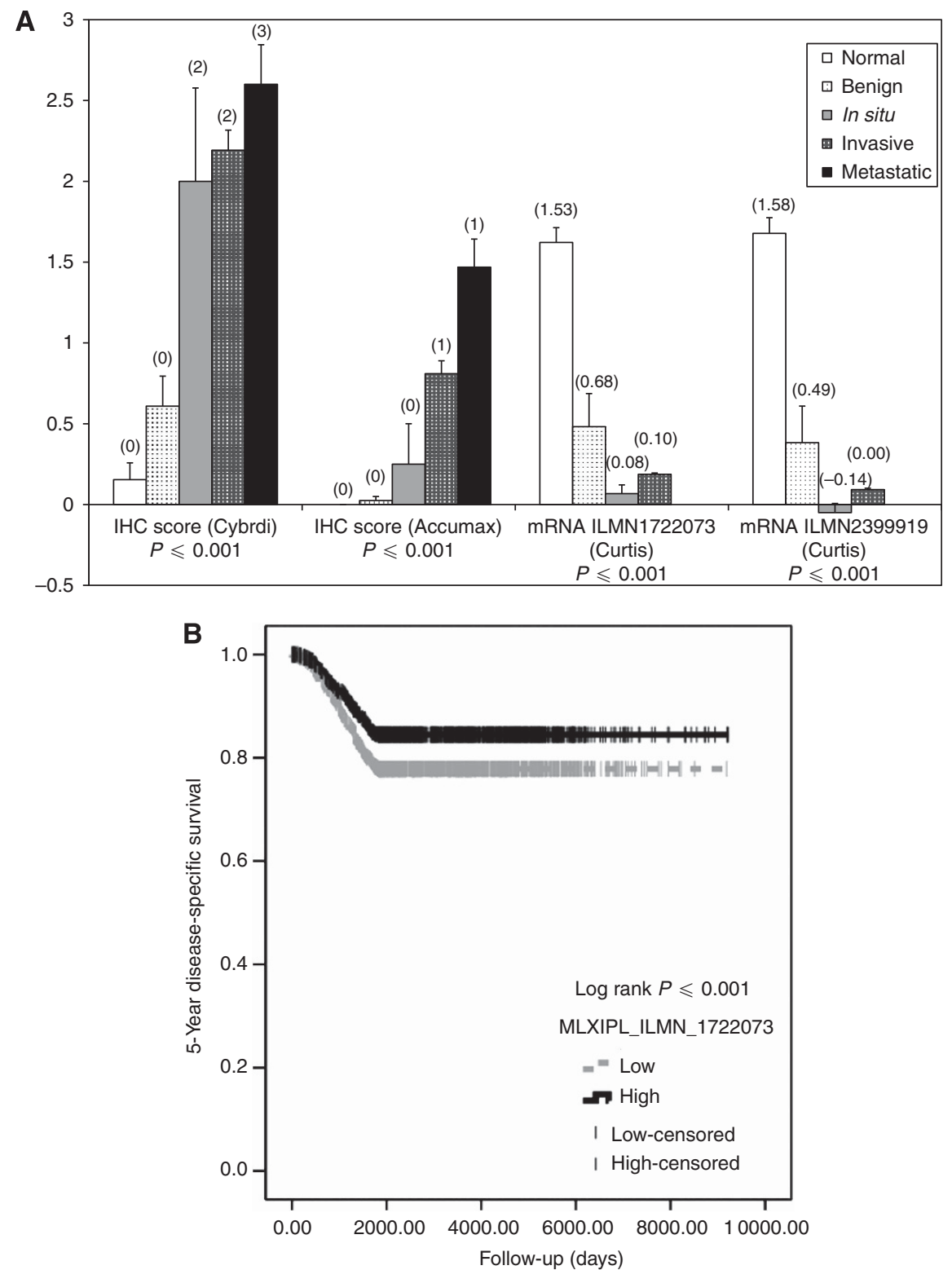

Figure 3. (A) There was a statistically significant (one-way ANOVA) trend whereby mean (median shown in brackets) ChREBP IHC score increased with malignant progression, consistent for both the Cybrdi and Accumax breast cancer progression arrays. In contrast, however, ChREBP mRNA expression data (log2 median-centred intensity) mined from the Curtis breast series showed a clear and significant decrease with malignant progression. Further analysis (B) of Curtis breast series showed high ( $\geqslant$ median 0.1265 log2 median-centred intensity) vs low ChREBP (MLXIPL ILMN_1722073) mRNA expression to be a significant predictor of 5-year disease-specific survival (log rank $P$-value also shown).

'downregulated' hypoxia scores, and as expected remained anticorrelated with mean and median 'upregulated' hypoxia scores (Figure 4). In the same data set, ChREBP mRNA was also anticorrelated with individual hypoxia-inducible genes such as CA9 $(r=-0.215, P=0.001)$ and $L D H A(r=-0.213, P=0.001)$ and, in contrast with the significant positive correlation shown by protein forms in the BRC 711 tissue microarray, ChREBP and Glut-1 mRNA in the Illumina data set were also significantly anticorrelated $(r=-0.212, P=0.001)$. A similar anticorrelation between ChREBP mRNA expression and these hypoxia-regulated genes was observed qualitatively in the Curtis breast series, as shown in heat maps generated via Oncomine (Supplementary Slides) and in an extended correlative analysis (Supplementary Table S1).

Correlates of ChREBP mRNA with ChREBP target genes. Jeong et al (2011) carried out a comprehensive ChIP sequence analysis of ChREBP target expression in human hepatocellular carcinoma HEPG2 cells and we used these to analyse the human breast cancer data sets. Table 1 shows the correlative relationships existing within the Oxf-IJB (Illumina) series of 232 patients between the level of ChREBP mRNA and that of its target genes previously identified by Jeong et al (2011). This was with the aim of highlighting any functional similarities or differences in the nature of ChREBP signalling in the hepatocellular carcinoma cell line used by Jeong et al (2011) and breast cancer. ChREBP mRNA expression, mined from the Illumina data set, correlated positively with the expression of all genes identified in the study of Jeong et al (2011) as upregulated by ChREBP apart from pyruvate dehydrogenase kinase 2 (PDK2). For genes identified as downregulated by ChREBP, although ChREBP mRNA mined from the Illumina data set mostly showed concordant negative regulation of these target genes, there was a positive correlation with the expression of 

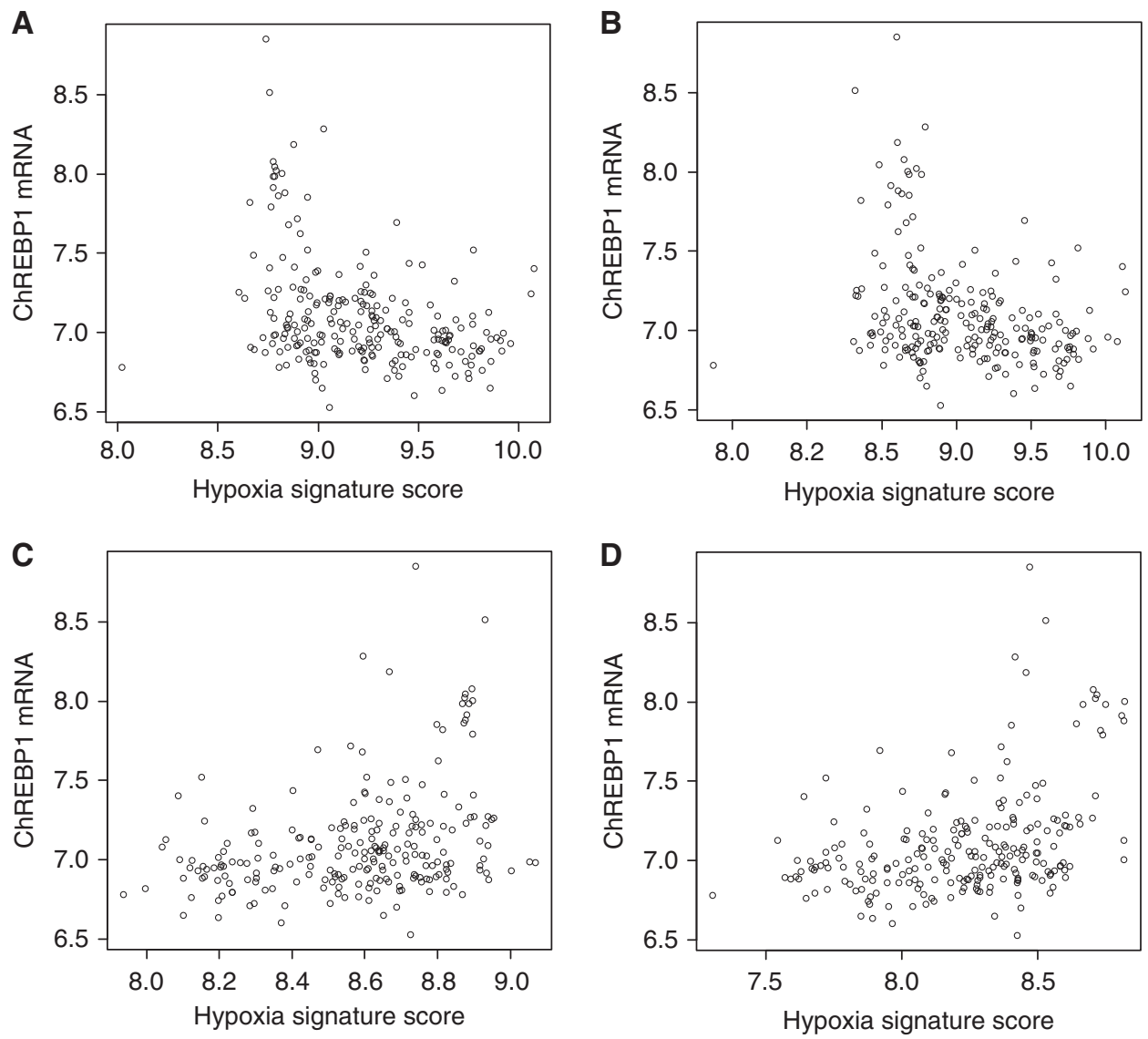

Figure 4. Scatter plots illustrating negative Spearman's rank correlations between ChREBP mRNA expression and (A) mean 'upregulated' hypoxia score $(r=-0.343, P=8.279 \mathrm{e}-08, n=232)$; $(B)$ median 'upregulated' hypoxia score $(r=-0.31, P=8.449 \mathrm{e}-07, n=232)$; and positive correlations with (C and D) mean $(r=0.318, P=7.268 \mathrm{e}-07, n=232)$ and median $(r=0.378, P=2.594 \mathrm{e}-09, n=232)$ 'downregulated' hypoxia scores (obtained from the Illumina Array data set from extended Oxf-IJB series).

Table 1. Spearman's rank correlations between carbohydrate response element-binding protein (ChREBP) and target genes previously identified by Jeong et al (2011), drawn from mRNA data mined from the breast cancer ( $n=232)$ Oxf-IJB [Illumina] data set

\begin{tabular}{|c|c|c|c|}
\hline Gene symbol & Description & Gene ID & $\boldsymbol{R}$ (P-value) Oxf-IJB [Illumina] data set \\
\hline \multirow[t]{2}{*}{$P K L R$} & Pyruvate kinase, liver and RBC & NM_181871 & [GI_32967596-A] $0.0453(0.492)$ \\
\hline & & & [GI_32967596-I] 0.0247 (0.709) \\
\hline SREBP1C or SREBF1 & Sterol-regulatory element-binding transcription factor 1 & NM_001005291 & $0.00517(0.938)$ \\
\hline DGAT2 & Diacylglycerol O-acyltransferase homolog 2 (mouse) & NM_032564 & $0.487(3.141 \mathrm{e}-15)$ \\
\hline PDK2 & Pyruvate dehydrogenase kinase, isozyme 2 & NM_002611 & $-0.137(0.0370)$ \\
\hline SCD1 & Stearoyl-CoA desaturase ( $\delta$-9-desaturase) & NM_005063 & $0.208(0.00147)$ \\
\hline GPDI or GPD1 & Glycerol-3-phosphate dehydrogenase 1 (soluble) & NM_005276 & $0.595(2.2 \mathrm{e}-16)$ \\
\hline TXNIP & Thioredoxin interacting protein & NM_006472 & $0.465(7.58 e-14)$ \\
\hline INHBE & Inhibin, $\beta \mathrm{E}$ & NM_031479 & $-0.154(0.0189)$ \\
\hline SLC6A9 & Solute carrier family 6 (neurotransmitter transporter, glycine), member 9 & NM_006934 & $-0.0866(0.189)$ \\
\hline PEPCK or PCK1 & Phosphoenolpyruvate carboxykinase 1 (soluble) & NM_002591 & $0.565(<2.2 \mathrm{e}-16)$ \\
\hline G6Pase or G6PC & Glucose-6-phosphatase, catalytic subunit & NM_000151 & $0.0237(0.720)$ \\
\hline TRIB3 or C20orf97 & Tribbles homolog 3 (Drosophila) & NM_021158 & $-0.224(0.000573)$ \\
\hline
\end{tabular}

phosphoenolpyruvate carboxykinase (PEPCK). The distributions of these data in relation to median expression of all mRNAs in the Illumina data set are presented as box-andwhisker plots in Figure 5. A strongly significant positive correlation between ChREBP and PEPCK was also found in the analysis of the Curtis breast series (Supplementary Table S1).

\section{DISCUSSION}

So far, there has been limited investigation of a possible role for ChREBP in tumour pathology or as a biomarker of malignancy. One previous study has shown no relationship between single- 
A

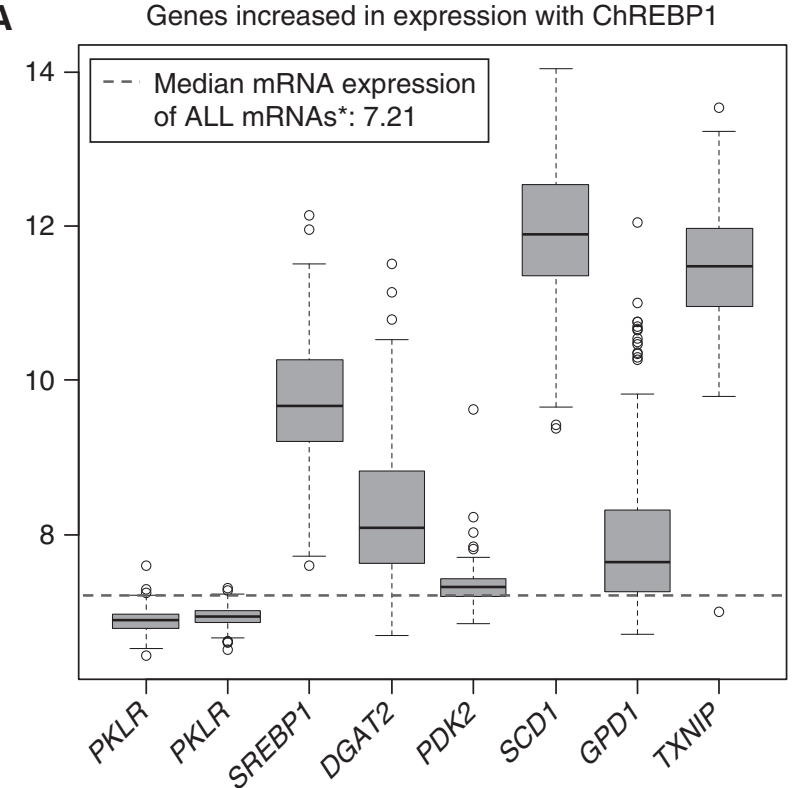

B

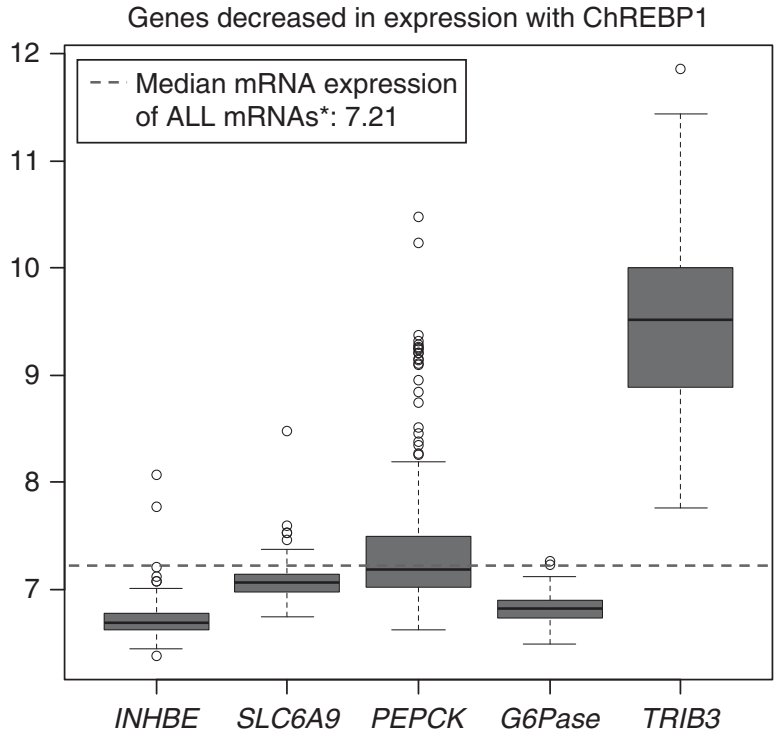

Figure 5. Box plot illustrating the distribution (median, upper, lower, 25th, 75th percentiles and outliers) of mRNA expression (log2 mediancentred intensity) of genes identified previously as ChREBP targets upregulated (A) and downregulated (B) in the study of Jeong et al (2011), mined from the breast cancer Oxf-IJB (Illumina) data set $(n=232)$. Correlations with PKLR (puruvate kinase) were drawn from data mined using both the (GI_32967596-A) and (GI_32967596-A) identifiers (see Table 1 for correlations and $P$-values).

nucleotide polymorphism of either ChREBP or FASN and breast cancer risk (Campa et al, 2009). The differential staining between normal adjacent and malignant tissue, as well as the clear increase in de novo expression of ChREBP protein with malignant progression, therefore suggests that local rather than genomic changes in ChREBP may have a role in driving tumourigenesis. It is puzzling that the majority of ChREBP expression appeared to be the cytoplasmic, phosphorylated and therefore inactive transcription factor. However, ChREBP exists in multiple isoforms that might vary in their tissue and tumour specificity as well as their up- and downstream regulatory effects. One recent study identified a regulatory path existing between the canonical ChREBP $\alpha$ isoform and $\operatorname{ChREBP} \beta$, where $\operatorname{ChREBP} \beta$ is induced by $\operatorname{ChREBP} \alpha$ via an alternative promoter in adipose tissue and predicts insulin sensitivity. This suggests that there might be not only an element of cross-talk between malignant breast and surrounding normal adipose tissue, but also that de novo synthesis of ChREBP may be partially self-regulated and critical to the changes in insulin-signalling that are a feature of breast tumour pathology and linked with cell proliferation and survival. The ChREBP association with insulin signalling and the correlation we have observed with Glut-1 may also determine how it interacts with important breast tumourigenic pathways such as the ER, epidermal growth factor receptor (EGFR), the insulin-like growth factor (IGF) systems and the mammalian target of rapamycin (mTOR) pathways (Martin and Baxter, 2007; Weichhaus et al, 2012; Yang and Yee, 2012).

Relationship between ChREBP protein and mRNA expression. The major function of ChREBP is the regulation of lipogenesis. However, HIF-1 and ChREBP share a function; to carry out their respective control of catabolic and anabolic pathways, they upregulate the expression of glycolytic genes. This increased rate of glycolysis is limited by a negative feedback mechanism mediated by the induction of TXNIP (thioredoxin interacting protein), a feedback mechanism common to both transcription factors ( $\mathrm{Yu}$ et al, 2010). Much of the understanding of the mechanistic effects of ChREBP has come about through studies involving cultured hepatocytes, where it has been observed that polyunsaturated but not saturated or monounsaturated fats suppress de novo synthesis of ChREBP, and that overexpression of ChREBP abolishes this effect (Dentin et al, 2005a). In addition, ChREBP is deactivated via activation of adenosine monophosphate-activated kinase (AMPK) that takes place in conditions where the AMP/ATP ratio is high, a consequence of increased diversion of the end products of glycolysis into fatty acid synthesis rather than oxidative phosphorylation (Hebbachi and Saggerson, 2013). Therefore, the existence of a negative feedback mechanism, although a complex one, where the induction of fatty acid synthesis by active nuclear ChREBP leads to eventual suppression of ChREBP mRNA synthesis, but where initial activation of existing ChREBP necessitates an increased rate of glucose uptake and metabolism, may exist. In breast tumours, this mechanism may be dependent upon the bioenergetic flux brought about by interacting populations of normoxic and hypoxic tumour and adipose tissue. The apparent inversion of the effects of ChREBP protein and mRNA on breast cancer progression, survival and the expression of hypoxiaregulated genes may be a reflection of such a mechanism.

Relationship between ChREBP and a normoxic malignant phenotype. Using a metabolomic approach, we have shown in a previous study that hypoxic conditions, particularly in the absence of an intact HIF-1 response, are countered by a rise in the AMP/ ATP ratio and the subsequent activation of the glycolytic enzyme phosphofructokinase (PFK-1) and AMPK (Golinska et al, 2011). The rise in the AMP/ATP ratio is also a key regulatory feature of AMPK-activated phosphorylation and inactivation of ChREBP, a feedback mechanism that downregulates ChREBP transcription in response to ChREBP-induced fatty acid synthesis (Kawaguchi et al, 2001; Fujii et al, 2006). The current findings highlight a correlation between ChREBP expression and the downregulated breast hypoxia signature, suggesting that ChREBP is expressed in normoxic conditions and a possible link with the suppression or downregulation of hypoxia and HIF-1-regulated genes. One explanation may be that, in hypoxic conditions, the activation of AMPK leads to downregulation of ChREBP and consequently fatty acid synthesis, favouring instead the B-oxidation pathway, where excess fatty acids may be broken down and fed back into the Krebs cycle. In normoxic conditions, however, the accumulation of pyruvate and subsequent conversion to acetyl CoA will feed into the first committed steps of ChREBP-inducible fatty acid synthesis. 
This hypothesis is supported by the evidence of an anticorrelation between ChREBP mRNA and PDK2 (Table 1) that may be indicative of PDK2 suppression brought about by aerobic conditions within the breast tumour microenvironment that will in turn favour the conversion of pyruvate into acetyl CoA via its downstream effect on the activation of pyruvate kinase (Contractor and Harris, 2012). Also, PDK2 increases accumulation of HIF-1 that may reflect the competing influences (Sun et al, 2009). The anticorrelation with the insulin modulator TRIB3, which is activated in hypoxic conditions and is associated with increased production of lactate and increased extracellular lactate levels, would also fit with this hypothesis (Mazzio et al, 2012). Interestingly, as with ChREBP in this study, TRIB3 in its protein and mRNA forms are oppositely associated with breast cancer prognosis. Despite TRIB3 being hypoxia inducible, this effect is linked to an inhibition of protein translation and redistribution of mRNA to areas within the cell that are less densely packed with ribosomes, which also takes place under anoxic conditions (Wennemers et al, 2012). Further work would be needed to determine whether a similar response explains the opposite association observed with ChREBP.

ChREBP as a mediator of alternative metabolic pathways in breast tumourigenesis. In hepatic tissue, ChREBP, together with sterol regulatory element-binding protein (SREBP), regulates glycolytic and lipogenic gene expression synergistically by inputting a response to increased glucose flux (Dentin et al, 2005b). Increased SREBP-mediated expression of FASN has been observed in hypoxia-exposed breast tumour cell lines alongside colocalisation of SREBP with the hypoxic regions of tumours (Furuta et al, 2008). This suggests that although SREBP and ChREBP share functional similarities, their dominance may depend upon the pattern of tumour oxygenation. Further evidence that the induction or suppression of ChREBP target genes may follow a different path in the breast tumour microenvironment from that of hepatic tissue is shown by the positive correlation between ChREBP mRNA and the gluconeogenic enzyme PEPCK that was suppressed in the presence of active ChREBP in the hepatocellular carcinoma cell line investigated by Jeong et al (2011). If, as the data of this study suggest, ChREBP is linked with normoxia, increased PEPCK expression in these conditions might correspond with the increased glucose demand of highly glycolytic but normoxic tumour tissue. This demand would potentially require supplementation via increased gluconeogenesis rather than via hypoxiainduced Glut-1 glucose transport. In contrast, in normal liver regeneration, PEPCK expression is itself increased via a hypoxiadriven HIF-1-dependent pathway (Tajima et al, 2009) unavailable in normoxic conditions.

In conclusion, our work suggests that the uptake of glucose to support a highly glycolytic phenotype in hypoxic conditions might be the primary role of glut- 1 in tumours, but a secondary role is to support the diversion of glycolytic end products into fatty acid synthesis in aerobic conditions. The resulting changes in tumour metabolism may then favour activation of downstream tumourigenic pathways via activation of ChREBP-induced target genes.

\section{CONFLICT OF INTEREST}

The authors declare no conflict of interest.

\section{REFERENCES}

Airley R, Evans A, Mobasheri A, Hewitt SM (2010) Glucose transporter Glut-1 is detectable in peri-necrotic regions in many human tumor types but not normal tissues: study using tissue microarrays. Ann Anat 192(3): 133-138.

Airley RE, Mobasheri A (2007) Hypoxic regulation of glucose transport, anaerobic metabolism and angiogenesis in cancer: novel pathways and targets for anticancer therapeutics. Chemotherapy 53(4): 233-256.

Annibaldi A, Widmann C (2010) Glucose metabolism in cancer cells. Curr Opin Clin Nutr Metab Care 13(4): 466-470.

Avril N, Menzel M, Dose J, Schelling M, Weber W, Janicke F, Nathrath W, Schwaiger M (2001) Glucose metabolism of breast cancer assessed by 18F-FDG PET: histologic and immunohistochemical tissue analysis. J Nucl Med 42(1): 9-16.

Bensinger SJ, Christofk HR (2012) New aspects of the Warburg effect in cancer cell biology. Semin Cell Dev Biol 23(4): 352-361.

Buffa FM, Camps C, Winchester L, Snell CE, Gee HE, Sheldon H, Taylor M, Harris AL, Ragoussis J (2011) microRNA-associated progression pathways and potential therapeutic targets identified by integrated mRNA and microRNA expression profiling in breast cancer. Cancer Res 71(17): 5635-5645.

Buffa FM, Harris AL, West CM, Miller CJ (2010) Large meta-analysis of multiple cancers reveals a common, compact and highly prognostic hypoxia metagene. Br J Cancer 102(2): 428-435.

Calle EE, Kaaks R (2004) Overweight, obesity and cancer: epidemiological evidence and proposed mechanisms. Nat Rev Cancer 4(8): 579-591.

Campa D, McKay J, Sinilnikova O, Husing A, Vogel U, Hansen RD, Overvad K, Witt PM, Clavel-Chapelon F, Boutron-Ruault MC, Chajes V, Rohrmann S, Chang-Claude J, Boeing H, Fisher E, Trichopoulou A, Trichopoulos D, Palli D, Villarini A, Sacerdote C, Mattiello A, Tumino R, Peeters PH, van Gils CH, Bas Bueno-de-Mesquita H, Lund E, Chirlaque MD, Sala N, Suarez LR, Barricarte A, Dorronsoro M, Sanchez MJ, Lenner P, Hallmans G, Tsilidis K, Bingham S, Khaw KT, Gallo V, Norat T, Riboli E, Rinaldi S, Lenoir G, Tavtigian SV, Canzian F, Kaaks R (2009) Genetic variation in genes of the fatty acid synthesis pathway and breast cancer risk. Breast Cancer Res Treat 118(3): 565-574.

Chen JL, Lucas JE, Schroeder T, Mori S, Wu J, Nevins J, Dewhirst M, West M, Chi JT (2008) The genomic analysis of lactic acidosis and acidosis response in human cancers. PLoS Genet 4(12): e1000293.

Contractor T, Harris CR (2012) p53 negatively regulates transcription of the pyruvate dehydrogenase kinase Pdk2. Cancer Res 72(2): 560-567.

Curtis C, Shah SP, Chin SF, Turashvili G, Rueda OM, Dunning MJ, Speed D, Lynch AG, Samarajiwa S, Yuan Y, Graf S, Ha G, Haffari G, Bashashati A, Russell R, McKinney S, Group M, Langerod A, Green A, Provenzano E, Wishart G, Pinder S, Watson P, Markowetz F, Murphy L, Ellis I, Purushotham A, Borresen-Dale AL, Brenton JD, Tavare S, Caldas C, Aparicio S (2012) The genomic and transcriptomic architecture of 2,000 breast tumours reveals novel subgroups. Nature 486(7403): 346-352.

Dang CV, Lewis BC, Dolde C, Dang G, Shim H (1997) Oncogenes in tumor metabolism, tumorigenesis, and apoptosis. J Bioenerg Biomembr 29(4): 345-354.

Dentin R, Benhamed F, Pegorier JP, Foufelle F, Viollet B, Vaulont S, Girard J, Postic C (2005a) Polyunsaturated fatty acids suppress glycolytic and lipogenic genes through the inhibition of ChREBP nuclear protein translocation. J Clin Invest 115(10): 2843-2854.

Dentin R, Girard J, Postic C (2005b) Carbohydrate responsive element binding protein (ChREBP) and sterol regulatory element binding protein-1c (SREBP-1c): two key regulators of glucose metabolism and lipid synthesis in liver. Biochimie 87(1): 81-86.

Evans A, Bates V, Troy H, Hewitt S, Holbeck S, Chung YL, Phillips R, Stubbs M, Griffiths J, Airley R (2008) Glut-1 as a therapeutic target: increased chemoresistance and HIF-1-independent link with cell turnover is revealed through COMPARE analysis and metabolomic studies. Cancer Chemother Pharmacol 61(3): 377-393.

Favaro E, Lord S, Harris AL, Buffa FM (2011) Gene expression and hypoxia in breast cancer. Genome Med 3(8): 55.

Fujii N, Jessen N, Goodyear LJ (2006) AMP-activated protein kinase and the regulation of glucose transport. Am J Physiol Endocrinol Metab 291(5): E867-E877.

Furuta E, Pai SK, Zhan R, Bandyopadhyay S, Watabe M, Mo YY, Hirota S, Hosobe S, Tsukada T, Miura K, Kamada S, Saito K, Iiizumi M, Liu W, Ericsson J, Watabe K (2008) Fatty acid synthase gene is up-regulated by hypoxia via activation of Akt and sterol regulatory element binding protein-1. Cancer Res 68(4): 1003-1011.

Golinska M, Troy H, Chung YL, McSheehy PM, Mayr M, Yin X, Ly L, Williams KJ, Airley RE, Harris AL, Latigo J, Perumal M, Aboagye EO, Perrett D, Stubbs M, Griffiths JR (2011) Adaptation to HIF-1 deficiency by 
upregulation of the AMP/ATP ratio and phosphofructokinase activation in hepatomas. BMC Cancer 11: 198.

Hebbachi A, Saggerson D (2013) Acute regulation of 5'-AMP-activated protein kinase by long-chain fatty acid, glucose and insulin in rat primary adipocytes. Biosci Rep 33(1): 71-82.

Jeong YS, Kim D, Lee YS, Kim HJ, Han JY, Im SS, Chong HK, Kwon JK, Cho YH, Kim WK, Osborne TF, Horton JD, Jun HS, Ahn YH, Ahn SM, Cha JY (2011) Integrated expression profiling and genome-wide analysis of ChREBP targets reveals the dual role for ChREBP in glucose-regulated gene expression. PLoS One 6(7): e22544.

Kawaguchi T, Takenoshita M, Kabashima T, Uyeda K (2001) Glucose and cAMP regulate the L-type pyruvate kinase gene by phosphorylation/ dephosphorylation of the carbohydrate response element binding protein. Proc Natl Acad Sci USA 98(24): 13710-13715.

Lorincz AM, Sukumar S (2006) Molecular links between obesity and breast cancer. Endocr Relat Cancer 13(2): 279-292.

Ma L, Tsatsos NG, Towle HC (2005) Direct role of ChREBP.Mlx in regulating hepatic glucose-responsive genes. J Biol Chem 280(12): 12019-12027.

Martin JL, Baxter RC (2007) Expression of insulin-like growth factor binding protein- 2 by MCF- 7 breast cancer cells is regulated through the phosphatidylinositol 3-kinase/AKT/mammalian target of rapamycin pathway. Endocrinology 148(5): 2532-2541.

Mazzio EA, Boukli N, Rivera N, Soliman KF (2012) Pericellular pH homeostasis is a primary function of the Warburg effect: inversion of metabolic systems to control lactate steady state in tumor cells. Cancer Sci 103(3): 422-432.

Postic C, Dentin R, Denechaud PD, Girard J (2007) ChREBP, a transcriptional regulator of glucose and lipid metabolism. Annu Rev Nutr 27: 179-192.

Scatena R, Bottoni P, Pontoglio A, Giardina B (2010) Revisiting the Warburg effect in cancer cells with proteomics. The emergence of new approaches to diagnosis, prognosis and therapy. Proteomics Clin Appl 4(2): 143-158.

Sun W, Zhou S, Chang SS, McFate T, Verma A, Califano JA (2009) Mitochondrial mutations contribute to HIFlalpha accumulation via increased reactive oxygen species and up-regulated pyruvate dehydrogenease kinase 2 in head and neck squamous cell carcinoma. Clin Cancer Res 15(2): 476-484.

Tajima T, Goda N, Fujiki N, Hishiki T, Nishiyama Y, Senoo-Matsuda N, Shimazu M, Soga T, Yoshimura Y, Johnson RS, Suematsu M (2009) HIF-1alpha is necessary to support gluconeogenesis during liver regeneration. Biochem Biophys Res Commun 387(4): 789-794.

Tong X, Zhao F, Mancuso A, Gruber JJ, Thompson CB (2009) The glucoseresponsive transcription factor ChREBP contributes to glucose-dependent anabolic synthesis and cell proliferation. Proc Natl Acad Sci USA 106(51): 21660-21665.

Uyeda K, Yamashita H, Kawaguchi T (2002) Carbohydrate responsive element-binding protein (ChREBP): a key regulator of glucose metabolism and fat storage. Biochem Pharmacol 63(12): 2075-2080.

Weichhaus M, Broom J, Wahle K, Bermano G (2012) A novel role for insulin resistance in the connection between obesity and postmenopausal breast cancer. Int J Oncol 41(2): 745-752.

Wennemers M, Bussink J, van den Beucken T, Sweep FC, Span PN (2012) Regulation of TRIB3 mRNA and protein in breast cancer. PLoS One 7(11): e49439.

Yang Y, Yee D (2012) Targeting insulin and insulin-like growth factor signaling in breast cancer. J Mammary Gland Biol Neoplasia 17: 251-261.

Young CD, Anderson SM (2008) Sugar and fat - that's where it's at: metabolic changes in tumors. Breast Cancer Res 10(1): 202.

Yu FX, Chai TF, He H, Hagen T, Luo Y (2010) Thioredoxin-interacting protein (Txnip) gene expression: sensing oxidative phosphorylation status and glycolytic rate. J Biol Chem 285(33): 25822-25830.

This work is published under the standard license to publish agreement. After 12 months the work will become freely available and the license terms will switch to a Creative Commons AttributionNonCommercial-Share Alike 3.0 Unported License.

Supplementary Information accompanies this paper on British Journal of Cancer website (http://www.nature.com/bjc) 